\title{
Evaluation of Electron Specific Absorbed Fractions in Organs of Digimouse Voxel Phantom Using Monte Carlo Simulation Code FLUKA
}

\author{
Sinha A. ${ }^{1 *}$, Singh N. ${ }^{2}$, Dixit B. M. ${ }^{1}$, Painuly N. K. ${ }^{2}$, Patni H. \\ K. ${ }^{3}$, Yadav A. ${ }^{4}$
}

\begin{abstract}
Background: For preclinical evaluations of radiopharmaceuticals, most studies are carried out on mice. Electron specific absorbed fractions (SAF) values have had vital role in the assessment of absorbed dose. In past studies, electron SAFs were given for limited source target pairs using older reports of human organ compositions.
\end{abstract}

Objective: Electron specific absorbed fraction values for monoenergetic electrons of energies $15,50,100,500,1000 \& 4000 \mathrm{keV}$ were evaluated for the Digimouse voxel phantom incorporated in Monte Carlo code FLUKA. From the latest report (International Commission on Radiological Protection ICRP) 110, organ compositions and densities were adopted.

Material and Methods: We have used the Digimouse voxel phantom which was incorporated in Monte Carlo code FLUKA. Simulation studies were performed using FLUKA. The organ sources considered in this study were lungs, skeleton, heart, bladder, testis, stomach, spleen, pancreas, liver, kidney, adrenal, eye and brain. The considered target organs were lungs, skeleton, heart, bladder, testis, stomach, spleen, pancreas, liver, kidney, adrenal and brain. Eye and brain were considered as target organs only for eye and brain as source organs.

Results: The electron SAF values for self-irradiation decreases with increasing electron energy. The electron SAF values for cross-irradiation are also found to be dependent on the electron energy and the geometries of source and target. Organ masses and electron SAF values are presented in tabular form.

Conclusion: The results of this study will be useful in evaluating the absorbed dose to various organs of mice similar in size to the present study.

\section{Keywords}

Electron specific Absorbed Fraction, Digimouse Voxel Phantom, Monte Carlo Simulation, FLUKA

\section{Introduction}

Do improve the estimation of organ doses, mice are frequently used. Mouse models can help to assess the potential interest of 1 new radiopharmaceuticals. Bio-distribution and radiation toxicity studies are performed on mice and based on success of these studies, human clinical trials will be carried out. The earliest methods adopted for estimation of electron specific absorbed fraction values involved using point kernel method [1-2] in conjunction with stylized phantoms [2-4] with mouse organs represented by ellipsoids, spheroids and cylinders. With time, approximate point kernel methods were replaced by full Monte Carlo simulations [2-6] using the particle transport which are

${ }^{1}$ Faculty of Physical Sciences, Shri Ramswaroop Memorial University,

Lucknow, India

${ }^{2}$ Department of Ra-

diotherapy, King George

Medical University,

Lucknow, India

${ }^{3}$ Bhabha Atomic Re-

search Center, Mumbai,

India

${ }^{4}$ Department of Ra-

diodiagnosis, Uttar

Pradesh Rural Institute

of Medical Sciences \&

Research, Saifai- Eta-

wah, India

*Corresponding author: A. Sinha

Faculty of Physical Sci-

ences, Shri Ramswaroop

Memorial University,

Lucknow, India

E-mail: ashutosh22jan@ gmail.com

Received: 30 January 2016 Accepted: 21 May 2016 
more accurate. Similarly, stylized phantoms were having the issue of non realistic representation of internal organs due to their simple mathematical form and non-overlapping organs. Mouse volume pixel (voxel) phantoms are the modern computational phantoms based on medical imaging of mouse. Most studies use older organ compositions based on International Commission on Radiological Protection (ICRP) publication number 23 [7] or International Commission on Radiation Units and Measurements (ICRU) report number 44 [8]. Previous studies also lack the aspect that electron specific absorbed fraction values are not provided for all source target pairs, which will be useful for computing all organ doses.

In this study, we present electron SAF as they are evaluated for the digimouse voxel phantom [9] using Monte carlo code FLUKA at six electron energies from $15 \mathrm{keV}$ to 4000 $\mathrm{keV}$. Electron SAF were evaluated and tabulated for all source target pairs as considered. The effect of various parameters on SAF values were also analyzed. The comparison between electron SAF values of this study and contemporary study which used voxelized phantom were also performed.

\section{Material And Methods}

\section{Digimouse Voxel Phantom in FLUKA Code}

The Digimouse voxel phantom used in this study which was downloaded [9] is a three- dimensional representation of whole body of a mouse. This phantom was developed by biomedical imaging group of the University of South California using medical image data such as X-ray, CT and color cryosection images of a normal nude male mouse. The phantom is a matrix of 380 columns, 992 rows and 208 slices or 78407680 voxels. The structures which are segmented and labeled with distinct organ identification numbers in this phantom are: medulla, cerebellum, olfactory bulbs, external cerebrum, striatum, rest of the brain, massetter muscles, eyes, lachrymal glands, heart, lungs, liver, stomach, spleen, pancreas, adrenal glands, kidneys, testes, bladder, skeleton and skin. The whole brain in this phantom consists of medulla, cerebellum, olfactory bulbs, external cerebrum, striatum and the rest of the brain.

Digimouse voxel phantom was converted into a suitable format for use in FLUKA code which was discussed in authors' previous work [10]. The figure of Digimouse voxel phantom was incorporated in FLUKA code (different planes) as shown in Figure 1. The elemental compositions and densities used for Monte Carlo simulation of Digimouse phantom are based on the most recent human anatomical data provided in ICRP publication number 110 [11]. ICRP 110 contains fifty three different organ compositions where various soft tissue compositions are based on ICRU 46 [12] and various skeleton tissue compositions are based on ICRP 70 [13].

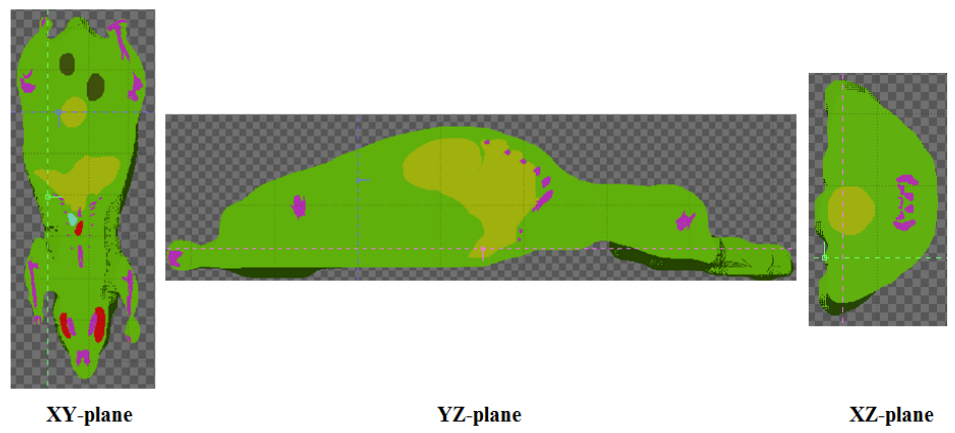

Figure 1: Digimouse Phantom incorporated in FLUKA code (different views) 
Simulations and Estimation

of Electron Specific Absorbed

Fractions

Masses of simulated organs (in Supporting documents) in phantom were calculated by multiplying the total volume of organs (product of number of voxels and volume of voxel and related density). The organ sources considered in the present study are: lungs, skeleton, heart, bladder, testis, stomach, spleen, pancreas, liver, kidney, adrenal, eye and brain. The target organs considered are: lungs, skeleton, heart, bladder, testis, stomach, spleen, pancreas, liver, kidney, adrenal and brain. As stated above, eye and brain were considered as the target only when eye and brain were source organs. Six electron energies $(15,50$, $100,500,1000$ and $4000 \mathrm{keV}$ ) were considered in these simulations. In FLUKA code, USERBIN card was used to evaluate the energy deposited in the target organs due to the activity in the source organ. The electron SAF value [14] used as a common parameter in our study and can be calculated by the following formula:

Average Energy Deposited in the Target Organ

$\mathrm{SAF}=$

Primary Energy Emitted in the Source Organ x mass of target organ

For a particular energy and source-target combination, $2 \times 10^{7}$ histories were run to reduce the relative error

(RE) in the estimated energy deposited in target organ due to the activity in source organ.

\section{Results and Discussion}

The computed electron SAF values (from equation 1) for various source-target combinations and their REs at different energies are presented in in Supporting documents. The reported electron SAF values have REs less than $10 \%$. The results of electron SAF values show that these values are dependent on the follow- ing factors:

Primary electron energy, mass of target organs, density of organ in case of self irradiation and effect of geometry in the case of cross-irradiation.

\section{Primary Electron Energy}

Because of low penetrating power of electrons, previous ICRP and MIRD $[15,16]$ assumption model supposed the electrons are fully absorbed in the source organ and electron AFs are recommended to be $1, \mathrm{AFs}$ and SAFs are recommended to be 0 when the source and target are different. From Figure 2, we can see that electrons have the ability to leave the source organ with electron energy above 0.5 $\mathrm{MeV}$ which proves that it is certainly not always appropriate to assume 100\% localized electron energy absorption.

Mass of Target Organs

We can see the dependence of mass of target organs in case of self-irradiation from Figure 2. The self-absorption SAFs agree with the inverse organ mass for electron energy. For self irradiation, liver has the smaller electron SAF values that is due to energy deposition per mass unit less for larger size organs. The organ with similar masses (difference $\sim 0.2 \%$ ) such as stomach and heart have very small differences $(<5 \%)$ in SAF values at all energies.

Effect of Density in case of Selfirradiation

Based on Figure 2, the difference between electron SAF values in lung and spleen, the organs having close masses, shows that the electron SAFs were dependent on organ density. Electron SAF values in lung and spleen show a variation of approximately $10 \%$ at lower energies and $80 \%$ at higher energies.

Effect of Geometry in case of Cross-irradiation

We can explain the independence of electron SAF values from mass of target organs 
in case of cross irradiation while taking spleen as a source organ. Figure 3 shows SAF values for organ cross-fire in adjacent organs such as stomach, pancreas, liver, kidney and heart with spleen as source organ. SAF values are different at all energies for stomach and heart (difference more than 100\%) regardless of having the same mass. We can conclude from these examples that SAF values for cross-irradiation depend on energy and geometrical fac- tors such as size of source and target.

\section{Comparison with Contemporary Stud-} ies using Digimouse Voxel Phantom

A comparison of electron AF values for self irradiation in liver reported by Mohammadi et al [17] and with those observed in the present study is shown in Figure 4. The difference in AF values of liver between our study and the reference study is less than $2 \%$ at $100 \mathrm{keV}$ as

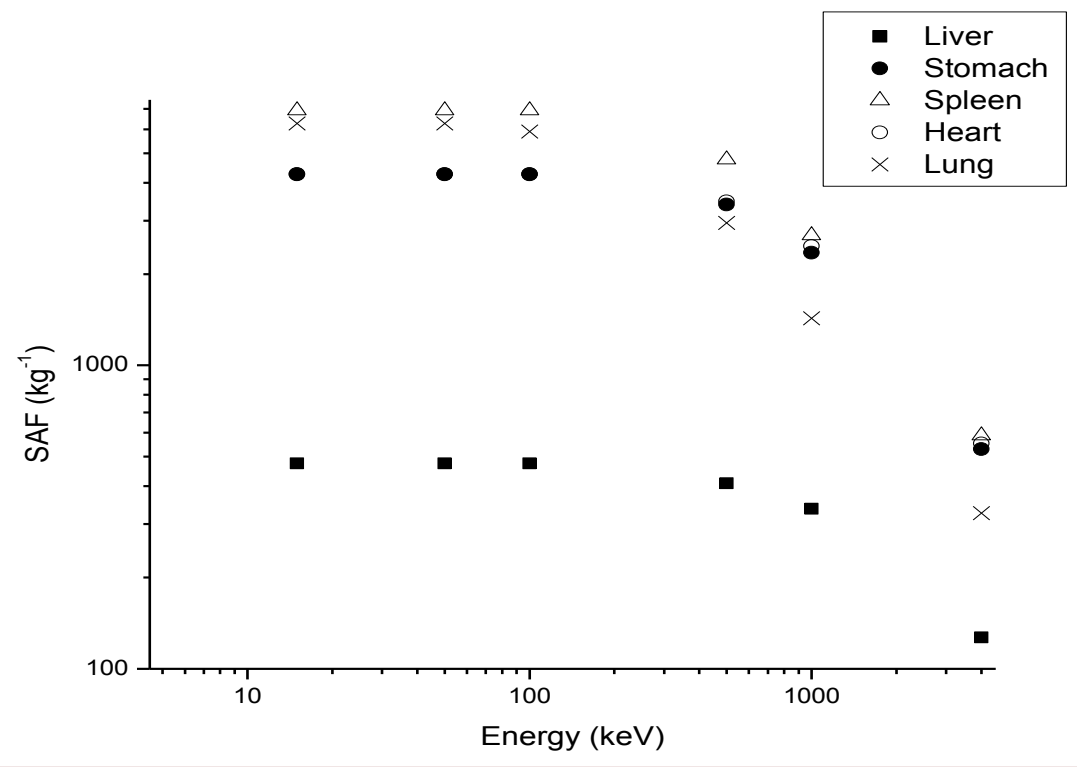

Figure 2: Self-irradiation in organs such as liver, stomach, spleen, heart and lung



Figure 3: Cross-irradiation in organs such as stomach, pancreas, liver, kidney and heart (source: spleen) 
well as very smaller differences at higher energies $(<2.69 \%)$. The AF values for cross irradiation in spleen, while taking liver as source organ between our study and the reference study, are shown in Figure 5, which have the minimum difference of $2.27 \%$ and maximum difference of $1 \%$.

\section{Conclusion}

The authors derived a new set of SAF val- ues for electrons at 15,50,100,500, 1000 and $4000 \mathrm{keV}$ in the organs of the Digimouse voxel phantom using FLUKA code. Studies on SAF values have used older standard human organ compositions and these values were for limited source target pairs. In this study, organ compositions and densities for photon SAF estimation in Digimouse voxelized phantom are based on ICRP publication number 110 . The organ sources were lungs, skeleton, heart,

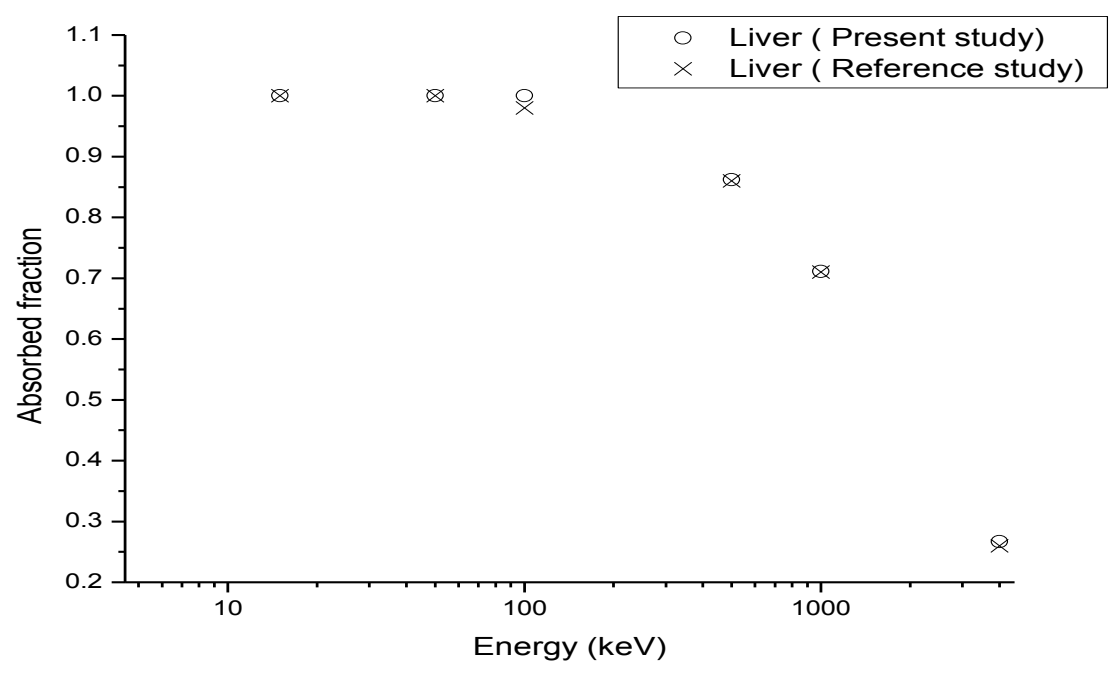

Figure 4: The comparison of absorbed fractions for self irradiation in liver between present study and Mohammadi et al. (reference study)

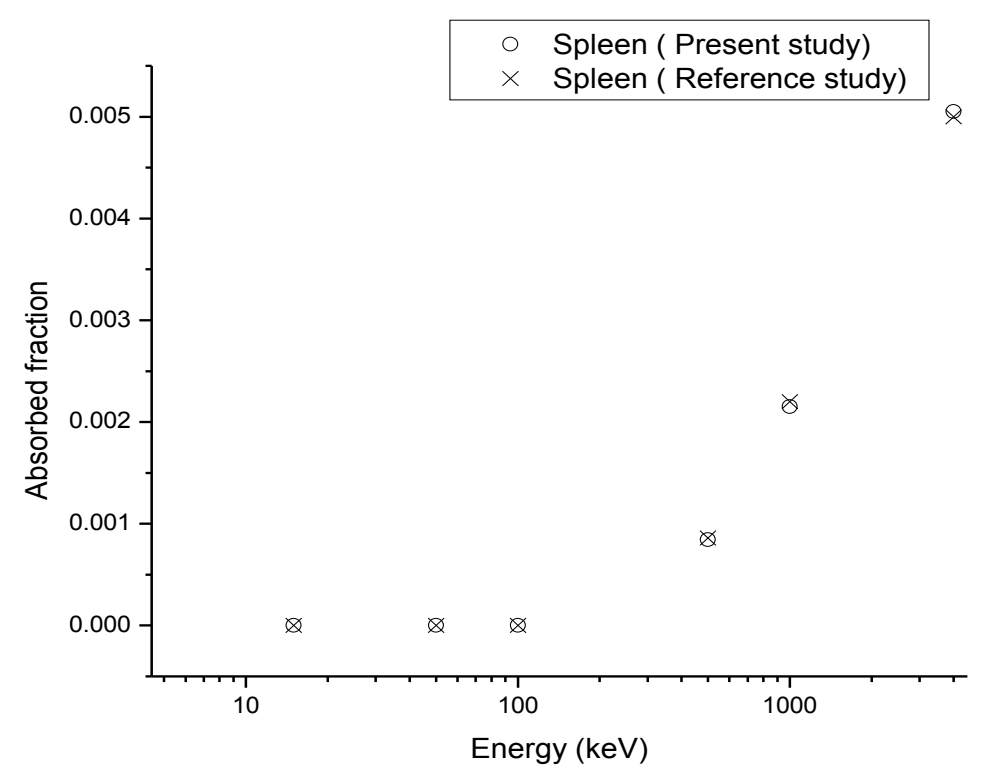

Figure 5: The comparison of absorbed fractions for cross irradiation in spleen between present study and Mohammadi et al. (reference study)[ Source-Liver] 
bladder, testis, stomach, spleen, pancreas, liver, kidney, adrenal, eye and brain. The target organs were lungs, skeleton, heart, bladder, testis, stomach, spleen, pancreas, liver, kidney, adrenal, eye and brain. The SAF values obtained from this study are in agreement with other studies which used Digmouse voxelized phantom. In case of self irradiation, electron SAF values depend on energy, mass and density of organs and for cross-irradiation, it depends on geometry of the organs.

\section{Acknowledgment}

The authors would like to thank Dr. Akram Mohammadi, National Institute of Radiological Sciences, Chiba- Japan for her guidance of this work.

\section{Conflict of Interest}

None

\section{References}

1. Hui TE, Fisher DR, Kuhn JA, Williams LE, Nourigat $C$, Badger CC, et al. A mouse model for calculating cross-organ beta doses from yttrium-90-labeled immunoconjugates. Cancer. 1994;73:951-7. PubMed PMID: 8306284.

2. Kolbert KS, Watson T, Matei $C, X u S$, Koutcher JA, Sgouros G. Murine $S$ factors for liver, spleen, and kidney. J Nucl Med. 2003;44:784-91. PubMed PMID: 12732681.

3. Hindorf C, Ljungberg M, Strand SE. Evaluation of parameters influencing $S$ values in mouse dosimetry. J Nucl Med. 2004;45:1960-5. PubMed PMID: 15534069.

4. Stabin MG, Peterson TE, Holburn GE, Emmons MA. Voxel-based mouse and rat models for internal dose calculations. J Nucl Med. 2006;47:655-9. PubMed PMID: 16595500.

5. Bitar A, Lisbona A, Thedrez P, Sai Maurel C, Le Forestier $D$, Barbet $J$, et al. A voxel-based mouse for internal dose calculations using Monte Carlo simulations (MCNP). Phys Med Biol. 2007;52:1013-25. doi.org/10.1088/0031-9155/52/4/010. PubMed PMID: 17264367.

6. Taschereau R, Chatziioannou AF. Monte Carlo simulations of absorbed dose in a mouse phantom from 18-fluorine compounds. Med Phys. 2007;34:1026-
36. doi.org/10.1118/1.2558115. PubMed PMID: 17441249. PubMed PMCID: 3006169.

7. Snyder W. Report of the Task Group on Reference Man: Prepared by a Task Group of Committee 2 of the International Commission on Radiological Protection: Adopted by the Commission in Oct. 1974: Pergamon; 1975.

8. ICRU, II. Tissue Substitutes in Radiation Dosimetry and Measurement. International Commission on Radiation Units and Measurements. 1989.

9. Dogdas B, Stout D, Chatziioannou AF, Leahy RM. Digimouse: a 3D whole body mouse atlas from CT and cryosection data. Phys Med Biol. 2007;52:57787. doi.org/10.1088/0031-9155/52/3/003. PubMed PMID: 17228106 . PubMed PMCID: 3006167.

10. Sinha A, Patni H, Dixit B, Painuly N, Singh N. Estimation of Photon Specific Absorbed Fractions in Digimouse Voxel Phantom using Monte Carlo Simulation Code FLUKA. Journal of Biomedical Physics and Engineering. 2015.

11. Menzel HG, Clement C, DeLuca P. ICRP Publication 110. Realistic reference phantoms: an ICRP/ ICRU joint effort. A report of adult reference computational phantoms. Ann ICRP. 2009;39:1-164. PubMed PMID: 19897132.

12. Photon E. Proton and Neutron Interaction Data for Body Tissues. ICRU report. 1992;46:13.

13. ICorpC. Basic Anatomical and Physiological Data for Use in Radiological Protection: the Skeleton: A Report of a Task Group of Committee 2 of the International Commission on Radiological Protection Adopted by the Commission in July 1994: Pergamon Press; 1995.

14. Patni HK, Akar DK, Nadar MY, Ghare VP, Rao DD, Sarkar PK. Estimation of specific absorbed fractions for selected organs due to photons emitted by activity deposited in the human respiratory tract using ICRP/ICRU male voxel phantom in FLUKA. Radiat Prot Dosimetry. 2013;153:3246. doi.org/10.1093/rpd/ncs087. PubMed PMID: 22645381.

15. Vennart J. Limits for intakes of radionuclides by workers. Practical Implications. 1979:175.

16. Synder WS, Ford MR, Warner GG. Estimates of Specific absorbed fractions for photon sources uniformly distributed in various organs of a Heterogeneous phantom. New York: Society of Nuclear Medicine; 1978. pp. 1-67.

17. Mohammadi A, Kinase S. Electron absorbed fractions and $S$ values in a mouse voxel phantom. Radioisotopes. 2011;60:505-12. doi.org/10.3769/ radioisotopes.60.505. 